\title{
Alta temprana y reconsultas del binomio madre-hijo en una institución de salud, Cali 2018
}

\author{
Early discharge and re-consultation of mother-child pair in a health \\ institution, Cali 2018
}

\author{
Victoria Bunzel Correa ${ }^{1,2}$, Antonio José Tascón ${ }^{3}$, Nora H. Riani-Llano ${ }^{3}$, Lina Maria \\ Ibáñez-Correa ${ }^{1,2,3}$, Robinson Pacheco López ${ }^{1,2}$ \\ ${ }^{1}$ Grupo de Investigación en Epidemiología y Servicios, Universidad Libre, Cali, \\ Colombia \\ ${ }^{2}$ Grupo Interdisciplinario de Investigación en Epidemiología y Salud Pública, \\ Universidad Libre, Cali, Colombia \\ ${ }^{3}$ Grupo de Investigación Clinver, Clínica Versalles S.A., Cali, Colombia
}

\section{Resumen}

Introducción: El tiempo de estancia hospitalaria del binomio madre-hijo luego del parto ha presentado cambios significativos. Antes, el binomio permanecía de 4 a 5 días luego del parto y en la actualidad ese tiempo fluctúa entre 24 a 48 horas. Existe la preocupación sobre si es un factor asociado al reingreso hospitalario del binomio madreHijo debido a complicaciones no detectables en la corta estancia.

Objetivo: Determinar la frecuencia de alta temprana y su relación con la seguridad del binomio madre-hijo en una clínica de alta complejidad de Cali, Colombia; de abril a junio de 2018.

Materiales y métodos: Se realizó un estudio observacional descriptivo longitudinal de binomios madres-hijos(as) atendidos en una IPS de Cali, de abril a junio de 2018.

Resultados: Se evaluaron 737 registros de la historia clínica correspondientes a binomios madre-hijo que recibieron su atención de parto en una institución de alta complejidad en la ciudad de Cali, y se encontró una frecuencia de reconsulta para el recién nacido de $18,31 \%$ donde las principales causas fueron problemas respiratorios, ictericia y problemas digestivos. Los factores asociados a la reconsulta del recién nacido y el binomio madre hijo fueron la escolaridad de la madre (2,02 IC 1,20-338) y el alta temprana (1,77 IC 1,03-3,02).

Conclusiones: Para el recién nacido el alta temprana mostró una frecuencia similar a la reportada en algunos estudios además de ser, al parecer, un factor asociado a la reconsulta; sin embargo, existen criterios clínicos que pueden ser implementados antes del alta para minimizar el riesgo de reconsulta.

Fecha de envío: 01/03/2020

Fecha de aceptación: 07/03/2021 
Palabras clave: Alta temprana postparto; Binomio madre-hijo; Readmisión del paciente; Seguridad.

\begin{abstract}
Background: The length of hospital stay of the mother-child binomial after delivery has presented significant changes. Previously, the binomial stayed 4 to 5 days after delivery and currently this time fluctuates between 24 to 48 hours. There is concern as to whether this is a factor associated with hospital readmission of the mother-child pair due to complications not detectable during the short stay. The objective is to determine the frequency of early discharge and its relationship with the safety of the mother-child binomial in a high complexity clinic in Cali, Colombia from April to June 2018.

Materials and methods: We conducted a longitudinal descriptive observational study of mother-child binomials attended in an IPS of Cali, from April to June 2018.

Results: 737 clinical history records corresponding to mother-child binomials who received their delivery care in a high complexity institution in the city of Cali. They were evaluated and a frequency of reconsultation for the newborn of $18.31 \%$ was found, where the main causes were: respiratory problems, jaundice and digestive problems. Factors associated with newborn and mother-child binomial reconsultation were the mother's schooling (2.02 CI 1.20-338) and early discharge (1.77 CI 1.03-3.02).

Conclusions: For the newborn, early discharge showed a frequency similar to that reported in some studies in addition to being, apparently, a factor associated with reconsultation; however, there are clinical criteria that can be implemented prior to discharge to minimize the risk of reconsultation.
\end{abstract}

Keywords: Early postpartum discharge; Mother-child binomial; Patient readmission; Safety.

\section{Introducción}

El tiempo de estancia hospitalaria del binomio madre-hijo, luego del parto vaginal no complicado, se ha reducido significativamente gracias al desarrollo prácticas médicas más seguras, avances en la tecnología biomédica y estándares de calidad más estrictos(1). Sin embargo, no existe un consenso sobre el punto de corte de la estancia hospitalaria adecuada para un egreso seguro del binomio.
Algunos autores refieren que el alta temprana connota beneficios clínicos como menor riesgo de infecciones asociadas a la atención en Salud (IAAS) y consecuentemente menor morbimortalidad, mayor éxito de la lactancia materna exclusiva, mejor calidad de vida, fortalecimiento del vínculo madre-hijo, autoconfianza de la madre y disminución del riesgo de depresión materna(2)(3)(4). En contraposición, otros autores resaltan algunos efectos negativos para el binomio relacionados 
con mayor frecuencia de reconsultas, derivadas de la disminución de la oportunidad de los diagnósticos de eventos latentes, complicaciones relacionadas a la atención del parto y por lo tanto mayor costo para el sistema $(5)(6)$.

La Asociación Americana de Pediatría (AAP) recomienda una estancia hospitalaria de al menos 48 horas, en la cual se debe evaluar 15 criterios clínicos del recién nacido para un egreso seguro y a la madre su condición clínica y psicosocial además del criterio del médico tratante. También recomienda que el alta del binomio madrehijo deberá ser simultáneo para asegurar el fortalecimiento de su vínculo y el éxito de la lactancia materna(6).

En Colombia, la guía de práctica clínica (2013) para la prevención, detección temprana y tratamiento de las complicaciones del embarazo, parto y puerperio, sugiere que todos los partos deben ser institucionales y que el tiempo de hospitalización posparto debe ser de al menos 48 horas y recomienda este mismo periodo de seguimiento estricto al binomio madre-hijo en aquellos casos de egresos más tempranos(7). De otro lado, la resolución 429 de 2016 del Ministerio de Salud de Colombia reglamenta la gestión del riesgo del binomio a través de la implementación de la ruta de atención integral materno perinatal. Esta es de obligatorio cumplimiento e indica que el egreso del binomio madrehijo luego de un parto vaginal sin complicaciones no debe darse a las 24 horas. Sin embargo, en el suroccidente del país la oferta de camas en instituciones habilitadas para la atención del parto es limitada frente a la alta demanda de estos servicios, por lo que el giro cama es inferior a
24 horas.

Así, dada la variabilidad de la evidencia y las características de las instituciones de salud, esta investigación buscó evaluar la seguridad del alta temprana con respecto a las reconsultas, debido a complicaciones no detectables en la corta estancia, de las maternas y recién nacidos quienes egresaron antes de las 24 horas, en una clínica en Cali, Colombia durante 2018.

\section{Materiales y Métodos}

Se realizó un estudio observacional descriptivo longitudinal con un alcance analítico. La población de estudio estuvo conformada por registros de binomios madre-hijo a quienes se les atendió parto vaginal entre abril y junio de 2018, en una clínica especializada en atención materno infantil de Cali. Se incluyeron las madres de todas las edades que tuvieron parto vaginal sin complicaciones clínicas reportadas en la historia clínica y con al menos un control prenatal y un control posparto dentro de los siguientes 20 días al parto; así como un recién nacido sin reporte de complicaciones asociadas al embarazo o al parto. Se excluyeron todos los embarazos múltiples y recién nacidos cuyas madres no respondieron al seguimiento telefónico.

Aunque no se realizó un cálculo del tamaño de muestra, dado que se aplicaron los criterios de selección a todos los registros disponibles durante el periodo del estudio, se verificó el poder estadístico sobre los registros disponibles. Se usó el paquete estadístico open epi disponible en https://www.openepi.com/ SampleSize/SSCohort.htm bajo los si- 
guientes parámetros: índice de confianza $95 \%$, error estándar del $5 \%$, se obtuvo un poder estadístico del $99 \%$.

Del departamento de estadística de la Institución se obtuvieron los registros de las pacientes que tuvieron su atención de parto entre abril y junio de 2018 y se realizó la selección de los registros según los criterios de selección. Se definió como alta temprana todos los egresos realizados hasta las 24 horas después del parto y el grupo de comparación se definió con los egresos después de las 24 horas de la atención del parto. Para proteger la identidad de los binomios madre-hijo, se enmascaró el nombre de la madre y la identificación.

Adicionalmente, se realizó seguimiento a través de llamadas telefónicas para indagar por el estado de salud del binomio $\mathrm{y}$ por las reconsultas tanto de la madre como del recién nacido a cualquier centro hospitalario en los siguientes 40 días luego del alta posparto. Para aumentar la tasa de respuesta en las no respondedoras, se realizaron dos llamadas consecutivas y una tercera en otro día. Para el análisis de la reconsulta para el binomio madre-hijo se definió como la reconsulta de la madre, el recién nacido o los dos.

Toda la información social, demográfica y clínica fue consignada en una base de datos electrónica (Excel@ Windows 2010) construida para el estudio. Utilizando el software STATA14®, se realizó un análisis univariado, aplicando estadística descriptiva; a todas las variables numéricas se les evaluó la distribución de los datos a través de la prueba estadística Kolmogorov Smirnov. Las variables cuantitativas se resumieron a través de la mediana y rango intercuartílico. Las variables nomi- nales se resumieron como porcentajes y se presentaron en tablas de frecuencias. Con la prueba de chi cuadrado o test exacto de Fisher se evaluó dependencia estadística entre las variables de exposición y desenlace. Para determinar las posibles relaciones entre las variables de exposición y la variable desenlace "Reconsulta" se usaron tablas de contingencia y la fuerza de asociación se midió a través de odds ratio (OR), con sus respectivos intervalos de confianza del $95 \%$. Para todas las pruebas estadísticas se consideró como significante valores de $p \leq 0,05$. Finalmente, para determinar el peso que cada variable de exposición aportó en la reconsulta, se construyeron modelos multivariables a través de regresión logística binomial, con las variables que en el análisis bivariable presentaron significancias estadísticas de $p \leq 0,25$. Los modelos se compararon con la prueba estadística razón de verosimilitudes y se seleccionó el modelo más parsimonioso.

Esta investigación fue aprobada por los comités de ética en investigación de la institución y el de la universidad. Los autores manifiestan no tener ningún conflicto de interés.

\section{Resultados}

Durante el periodo de estudio se evaluaron los criterios de selección a 1144 registros de pacientes y sus recién nacidos que tuvieron su atención del parto vaginal en la institución de estudio. Un total de $64,4 \%(n=737)$ registros cumplieron criterios de selección y se excluyeron 407 registros en total; 258 registros correspondían a madres con diagnósticos clíni- 


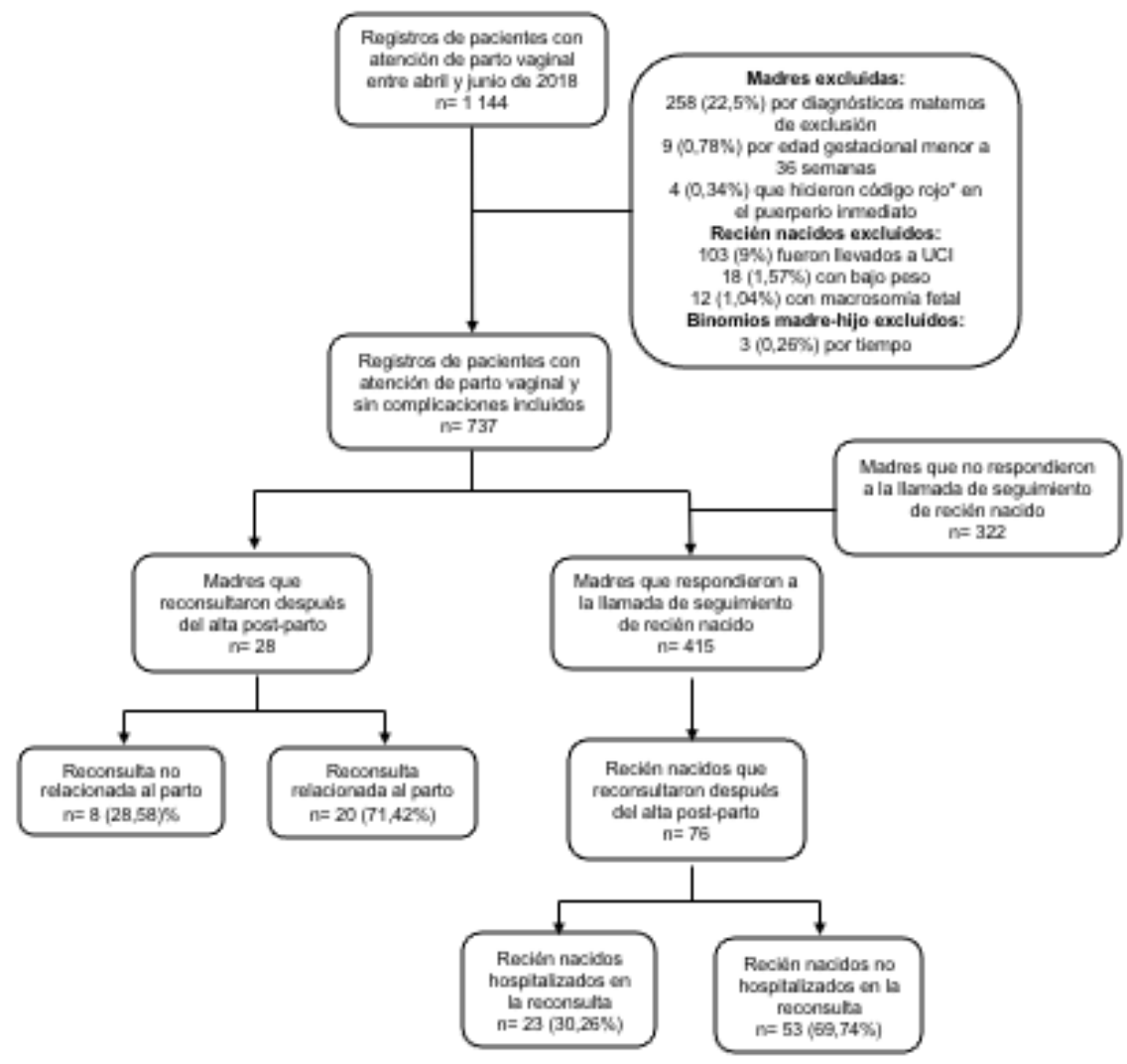

Figura 1: Diagrama para la selección y la clasificación de binomios madre-hijo.

${ }^{*}$ Código Rojo: Esquema de trabajo organizado que se ejecuta cuando se presenta una hemorragia obstétrica

cos de exclusión, 9 pertenecían a madres con gestaciones menores a 36 semanas, 4 correspondieron a madres que tuvieron código rojo luego del parto, 103 debido a que el recién nacido requirió ser llevado a la unidad de cuidado intensivo neonatal, 18 pertenecían a recién nacidos con bajo peso al nacer, 12 pertenecían a recién nacidos con macrosomia y 3 registros pertenecientes a binomios que tuvieron un tiempo de egreso prolongado debido a condiciones clínicas luego de parto. Los recién nacidos cuyas madres no respondieron el se- guimiento telefónico fueron excluidos para el análisis de reconsulta, por lo que quedaron un total de 415 recién nacidos (Figura 1). La frecuencia de reconsulta para el binomio madre-hijo fue de $13,7 \%(\mathrm{n}=101)$ $\mathrm{y}$ en las madres fue de $3,79 \%(\mathrm{n}=28)$, del cual $71,42 \%(n=20)$ fueron asociadas al parto. Para el recién nacido, el porcentaje de respuesta de la llamada de seguimiento de reconsulta fue $56,3 \% \quad(\mathrm{n}=415)$ y una frecuencia de reconsulta de 18,31\% $(\mathrm{n}=76)$; de los 76 recién nacidos que reconsultaron $68,42 \%(\mathrm{n}=52)$ habían sido 


\begin{tabular}{|c|c|c|c|c|c|}
\hline Característica & Descripción & $\begin{array}{l}\text { Total } \\
737)\end{array}(\mathrm{n}=$ & $\begin{array}{l}\text { Alta antes } \\
\text { de las } \\
24 \text { horas } \\
(n=396)\end{array}$ & $\begin{array}{lr}\text { Alta } & \text { des- } \\
\text { pués } & \text { de } \\
\text { las } & 24 \\
\text { horas } & \\
(\mathrm{n}=341)\end{array}$ & $\begin{array}{l}\text { Valor } \\
\text { p }\end{array}$ \\
\hline \multirow{2}{*}{$\begin{array}{l}\text { Edad en años de } \\
\text { la } \\
\text { madre* }\end{array}$} & \multirow{2}{*}{ Años } & 25,93 & 26 & 25 & \multirow{2}{*}{0,13} \\
\hline & & $(22-30)$ & $(22-30)$ & $(22-30)$ & \\
\hline \multirow{2}{*}{$\begin{array}{l}\text { Gestaciones de la } \\
\text { madre* }\end{array}$} & \multirow{2}{*}{ Número de embarazos } & 1,76 & 2 & 1 & \multirow{2}{*}{0,98} \\
\hline & & $(1-2)$ & $(1-2)$ & $(1-2)$ & \\
\hline \multirow{2}{*}{ Edad Gestacional* } & \multirow{2}{*}{ Número de semanas } & 39,04 & 39 & 39 & \\
\hline & & $(38-40)$ & $(38-40)$ & $(38-40)$ & \\
\hline \multirow{6}{*}{$\begin{array}{l}\text { Escolaridad de la } \\
\text { madre }\end{array}$} & $\begin{array}{l}\text { Básica primaria y se- } \\
\text { cundaria }\end{array}$ & $88(11,9)$ & 45 & 43 & \multirow{6}{*}{0,49} \\
\hline & $\begin{array}{l}\text { Media académica } \mathrm{y} \\
\text { Técnica }\end{array}$ & $321(43,5)$ & 166 & 155 & \\
\hline & $\begin{array}{l}\text { Técnica profesional y } \\
\text { Tecnología }\end{array}$ & $233(31,6)$ & 131 & 102 & \\
\hline & Profesional & $89(12,08)$ & 51 & 38 & \\
\hline & Posgrado & $4(0,54)$ & 1 & 3 & \\
\hline & Sin información & $2(0,27)$ & 2 & 0 & \\
\hline \multirow{2}{*}{$\begin{array}{l}\text { Controles prena- } \\
\text { tales* }\end{array}$} & \multirow{2}{*}{$\begin{array}{l}\text { Número de controles } \\
\text { prenatales }\end{array}$} & 6 & 6 & 6 & \multirow{2}{*}{0,33} \\
\hline & & $(6-7)$ & $(6-7)$ & $(6-7)$ & \\
\hline \multirow{2}{*}{$\begin{array}{l}\text { Sexo del Recién } \\
\text { Nacido }\end{array}$} & Masculino & $360(48,85)$ & 198 & 162 & \multirow{2}{*}{0,50} \\
\hline & Femenino & $377(51,15)$ & 198 & 179 & \\
\hline \multirow{2}{*}{$\begin{array}{l}\text { Peso del Recién } \\
\text { Nacido* }\end{array}$} & \multirow{2}{*}{ Gramos } & 3263,75 & 3265 & 3230 & 0,31 \\
\hline & & $(2995-3503)$ & $(2999-3523)$ & $(2995-3485)$ & \\
\hline
\end{tabular}

Tabla 1: Características sociodemográficas de la población de estudio por grupo de comparación. * Medida resumen para las variables cuantitativas Mediana y Rango intercuartílico

dados de alta tempranamente (Figura 1).

\subsection{Características demográfi- cas de los binomios madre- hijo}

La mediana de edad de las madres fue de 25,93 años (RIC 22-30). Con respecto al nivel educativo, $38,26 \%(282 / 737)$ re- portaron haber cursado toda la secundaria y $24,02 \%(177 / 737)$ tenían formación técnica o profesional. En cuanto a la paridad, $50 \%$ de las madres reportaron tener más de un hijo (mediana 1,72 RIC 12). Para la edad gestacional la mediana fue de 39,04 semanas (RIC 38-40). Respecto a los controles prenatales, al $50 \%$ 
de las madres se les registró entre 6 y 7. Referente a los recién nacidos, $51,15 \%$ (377/737) fueron de sexo femenino; la mediana del peso fue de 3,263 gramos (RIC 2,995- 3,503). De los binomios madre-hijo, $46,27 \%$ (346/737) fueron egresados antes de las 24 horas después del parto (Tabla $1)$.

No se identificaron factores demográficos o clínicos relacionados al egreso de los binomios madre-hijo antes o después de las 24 horas post parto (Tabla 1).

Las causas de reconsulta más frecuentes para el recién nacido encontradas en esta investigación fueron, en primer lugar, problemas respiratorios $27,63 \% \quad(\mathrm{n}=21)$; seguido por ictericia, con un 22,36\% $(\mathrm{n}=17) ; \mathrm{y}$ como tercera causa más frecuente por problemas digestivos, con un $15,78 \%(\mathrm{n}=12)$. De los 76 recién nacidos que reconsultaron, $30,26 \%(\mathrm{n}=23)$ fueron hospitalizados por diferentes causas con una mediana de 10 días (RIC 3-10) y 52 habían sido dados de alta tempranamente (Tabla 2).

Sin embargo, es importante tener en cuenta que de los 415 recién nacidos donde la madre respondió el seguimiento telefónico, 239 de ellos habían sido dados de alta tempranamente por lo que solo $9,6 \%$ requirió hospitalización en la reconsulta.

En el análisis de las causas de reconsulta materna, esta investigación encontró que las más frecuentes son: el dolor, cólico y/o ardor en la zona del abdomen bajo, con un $28,57 \%(\mathrm{n}=8)$; y las causas no relacionadas al parto $28,57 \%(\mathrm{n}=8)$, tuvieron la misma frecuencia como causa de consulta. Las madres que debieron ser hospitalizadas durante la reconsulta después del parto fueron $21,42 \%(n=6)$ por diferentes causas y la mediana de días de hospitalización fue 2,6 (RIC 2-5) días (Tabla 2).

Con respecto a la reconsulta del binomio madre-hijo, las variables que mostraron relación estadística significante fueron: escolaridad de la madre cuando la escolaridad era técnica profesional y tecnológica (OR 4,20 [IC 95\%: 1,48 - 11,9], p-0,010) y alta temprana (OR 1,74 [IC 95\%: 1,12 - 2,71], p-0,012). Para la reconsulta del recién nacido las variables que mostraron asociación estadística significante fueron: número de controles prenatales (6 controles) (OR 1,21 [IC 95\%: $0,98-1,51], \mathrm{p}-0,075)$ y alta temprana (OR 1,76 [IC 95\%: 1,04 -2,99], p-0,036). Para la reconsulta de la madre, la variable que mostró significancia estadística fue el peso del recién nacido (OR 1,00 [IC 95\%: 0,99 - 1,00], p-0,056) (Tabla 3).

El análisis multivariado reveló que los factores asociados a la reconsulta del binomio madre-hijo fueron la escolaridad de la madre y el alta temprana (Tabla 4). Adicionalmente, no mostró factores relacionados a la reconsulta de la madre, mientras que para la reconsulta del recién nacido (OR ajustado), la escolaridad de la madre en un nivel de educación de técnico profesional a posgrado y el alta temprana sí lo estaban (Tabla 4).

\section{Discusión}

Esta investigación evaluó la frecuencia del alta temprana y la seguridad para el binomio madre-hijo a quienes se les atendió el parto vaginal sin complicaciones para ninguno de los dos. Nuestros hallazgos encontraron una frecuencia de alta tem- 


\begin{tabular}{|c|c|c|}
\hline Motivo de Reconsulta & $\begin{array}{l}\text { Frecuencia } \\
\%\end{array}$ & $\begin{array}{l}\text { Hospitalización } \\
\%\end{array}$ \\
\hline \multicolumn{3}{|l|}{ Recién Nacido } \\
\hline 1: Problemas respiratorios & $21(27,63)$ & $7(9,21)$ \\
\hline 2: Brote & $1(1,31)$ & $0(0,0)$ \\
\hline 3: cardiopatía & $1(1,31)$ & $1(1,31)$ \\
\hline 4: Problemas digestivos & $12(15,78)$ & $2(2,63)$ \\
\hline 5: Conjuntivitis & $4(5,26)$ & $0(0,0)$ \\
\hline 6: Fractura clavícula & $2(2,63)$ & $0(0,0)$ \\
\hline 7: Convulsión & $1(1,31)$ & $1(1,31)$ \\
\hline 8: Deshidratación & $1(1,31)$ & $0(0,0)$ \\
\hline 9: Fiebre & $5(6,57)$ & $3(3,94)$ \\
\hline 10: Hipoglicemia & $2(2,63)$ & $0(0,0)$ \\
\hline 11: Ictericia & $17(22,36)$ & $5(6,57)$ \\
\hline $\begin{array}{l}\text { 12: Infección y/o sangrado } \\
\text { ombligo }\end{array}$ & $5(6,57)$ & $0(0,0)$ \\
\hline 13: IVU* & $1(1,31)$ & $1(1,31)$ \\
\hline 14: Infección viral & $2(2,63)$ & $2(2,63)$ \\
\hline 15: Sangrado vaginal & $1(1,31)$ & $1(1,31)$ \\
\hline \multicolumn{3}{|l|}{ Materna } \\
\hline $\begin{array}{l}\text { 1: Dolor, cólico y/o ardor en } \\
\text { zona del abdomen bajo }\end{array}$ & $8(28,57)$ & $2(7,14)$ \\
\hline $\begin{array}{l}\text { 2: Trastorno mixto de ansie- } \\
\text { dad y depresión }\end{array}$ & $1(3,57)$ & $1(3,57)$ \\
\hline 3:HTA posparto** & $2(7,14)$ & $2(7,14)$ \\
\hline 4: Hemorroides & $1(3,57)$ & $0(0,0)$ \\
\hline $\begin{array}{l}\text { 5: Causas no relacionadas al } \\
\text { parto }\end{array}$ & $8(28,57)$ & $0(0,0)$ \\
\hline 6: Mastitis & $3(7,14)$ & $1(3,57)$ \\
\hline 7: Dehiscencia de sutura & $3(7,14)$ & $0(0,0)$ \\
\hline $\begin{array}{l}\text { 8: Retención de fragmentos } \\
\text { placenta }\end{array}$ & $1(3,57)$ & $0(0,0)$ \\
\hline 9: Fiebre & $1(3,57)$ & $0(0,0)$ \\
\hline
\end{tabular}

Tabla 2: Causas de reconsulta del recién nacido y de la materna.

*IVU: Infección de las vías urinarias. ${ }^{*} *$ HTA: Hipertensión arterial

prana de $53,7 \%$. Esta es similar a la reportada por Cardona y Cols, quienes rea- lizaron un estudio de una cohorte prospectiva de mujeres con seguimiento posparto 
Bunzel et al.

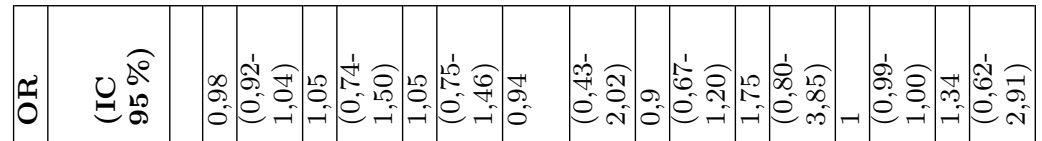

\begin{tabular}{|l|l|l|l|l|l|l|l|l|}
\hline$a$ & 8 & 0 & 0 \\
\hline
\end{tabular}

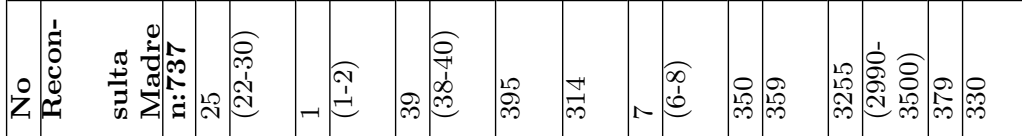

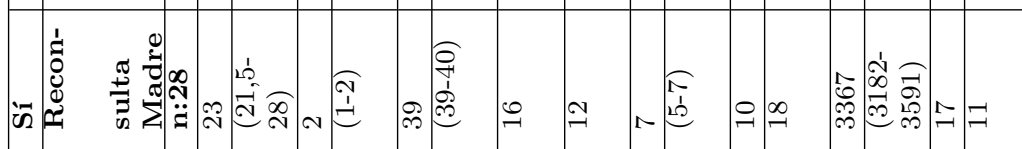

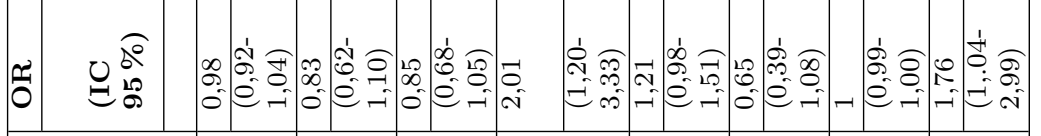

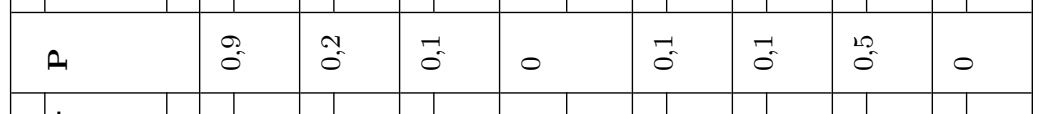

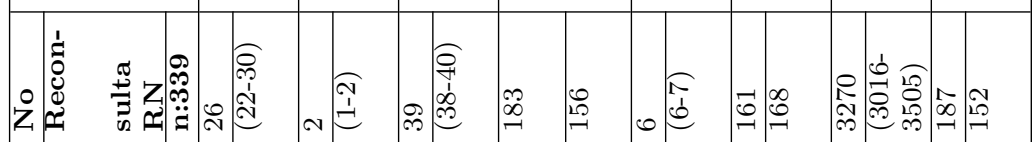

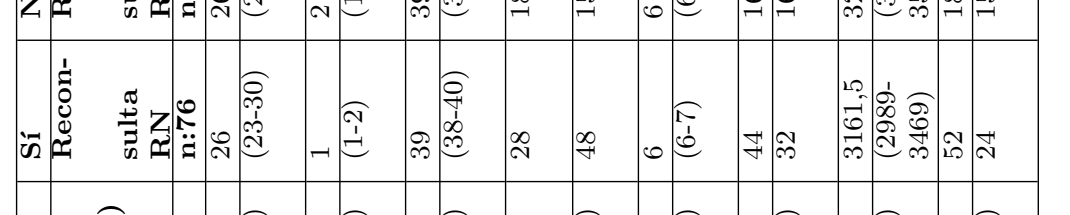

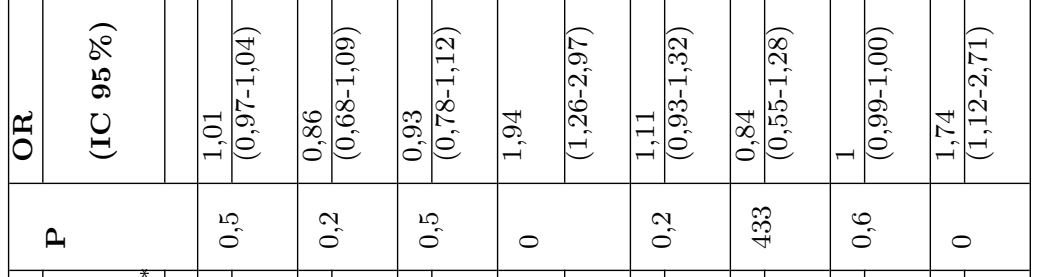

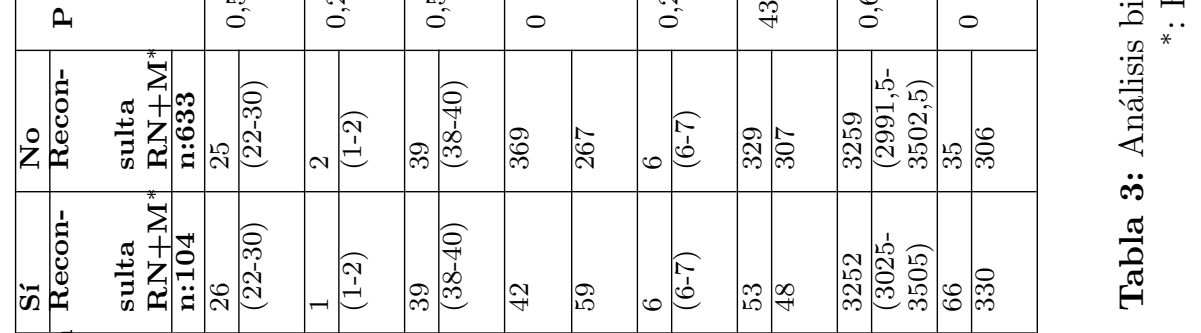

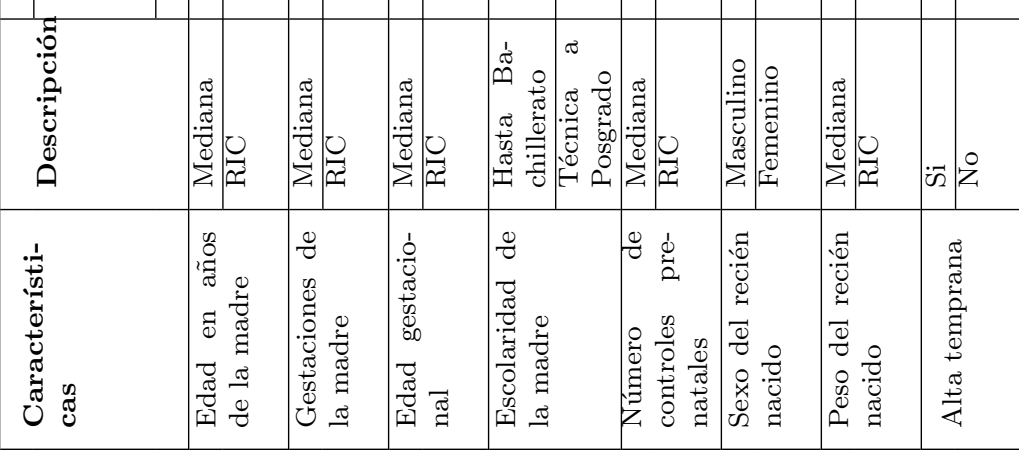




\begin{tabular}{|c|c|c|c|c|c|c|c|c|c|c|}
\hline $\begin{array}{l}\text { Característi- } \\
\text { ca }\end{array}$ & Descripción & $\mathrm{n}$ & $\begin{array}{l}\text { Sí } \\
\text { Reconsulta* } \\
(n=76)\end{array}$ & $\begin{array}{l}\text { No } \\
\text { Reconsulta* } \\
(n=339\end{array}$ & OR & IC $95 \%$ & $\mathbf{P}$ & $\begin{array}{l}\text { OR } \\
\text { ajus- } \\
\text { tado }\end{array}$ & IC $95 \%$ & $\mathbf{P}$ \\
\hline \multicolumn{11}{|c|}{ Entre variables de exposición y reconsulta recién nacido } \\
\hline \multirow[t]{2}{*}{$\begin{array}{l}\text { Escolaridad de } \\
\text { la madre }\end{array}$} & $\begin{array}{l}\text { Hasta Ba- } \\
\text { chillerato }\end{array}$ & 411 & 42 & 369 & \multirow[t]{2}{*}{1,94} & \multirow[t]{2}{*}{$1,26-2,97$} & \multirow[t]{2}{*}{0,002} & \multirow[t]{2}{*}{1,9} & \multirow[t]{2}{*}{$1,24-2,92$} & \multirow[t]{2}{*}{0,003} \\
\hline & $\begin{array}{l}\text { Técnica a } \\
\text { posgrado }\end{array}$ & 326 & 59 & 267 & & & & & & \\
\hline \multirow{2}{*}{ Alta temprana } & $\mathrm{Si}$ & 101 & 66 & 35 & \multirow{2}{*}{1,74} & \multirow{2}{*}{$1,12-2,71$} & \multirow{2}{*}{0,012} & \multirow{2}{*}{1,71} & \multirow{2}{*}{$1,1-2,66$} & \multirow{2}{*}{0,017} \\
\hline & No & 636 & 330 & 306 & & & & & & \\
\hline \multicolumn{11}{|c|}{ Entre variables de exposición y reconsulta recién nacido } \\
\hline \multirow[t]{2}{*}{ Escolaridad } & $\begin{array}{l}\text { Hasta ba- } \\
\text { chillerato }\end{array}$ & 211 & 28 & 183 & \multirow[t]{2}{*}{2,01} & \multirow[t]{2}{*}{$1,2-3,33$} & \multirow[t]{2}{*}{0,08} & \multirow[t]{2}{*}{2,02} & \multirow[t]{2}{*}{$1,20-3,38$} & \multirow[t]{2}{*}{0,007} \\
\hline & $\begin{array}{l}\text { Técnica a } \\
\text { posgrado }\end{array}$ & 204 & 48 & 156 & & & & & & \\
\hline \multirow{2}{*}{ Alta temprana } & $\mathrm{Si}$ & 239 & 52 & 187 & \multirow{2}{*}{1,76} & \multirow{2}{*}{$1,04-2,99$} & \multirow{2}{*}{0,036} & \multirow{2}{*}{1,77} & \multirow{2}{*}{$1,03-3,02$} & \multirow{2}{*}{0,035} \\
\hline & No & 176 & 24 & 152 & & & & & & \\
\hline
\end{tabular}

Tabla 4: Modelo múltiple de medidas de asociación entre variables de exposición y reconsulta binomio madre-hijo y del recién nacido.

$*$ : Reconsulta binomio.

con un total de 750 madres en una clínica de Medellín, donde la frecuencia de alta temprana antes de las 24 horas reportada fue de $51,7 \%$. Como en nuestro estudio, Cardona y Cols tampoco encontraron diferencias o factores clínicos o demográficos que determinaran el egreso hospitalario antes de las 24 horas; al parecer, el único determinante del egreso temprano serían trámites administrativos(3).

Un estudio de costo efectividad del alta temprana realizado por Rueda y Cols menciona que una de las razones por las que las Instituciones Prestadoras de Salud (IPS) egresan tempranamente a los binomios madre-hijo es para mejorar su eficiencia productiva(8). Por otro lado, la Guía de práctica clínica para la prevención, detección temprana y tratamiento de las complicaciones del embarazo parto o puerperio $(2013)(7)$ describe en la relación de la evidencia y la recomendación que la decisión del alta en el posparto tie- ne implicaciones sobre los recursos, bien para incrementar el número de camas o la disponibilidad de personal calificado. Sin embargo, recomienda que si el egreso se da tempranamente, debe realizarse un seguimiento a las 48 horas(7).

$\mathrm{Al}$ comparar la frecuencia de reconsulta luego del alta temprana del recién nacido de nuestro estudio, se encontró una frecuencia menor en el estudio Boulvain y Cols, que fue un ensayo aleatorizado sobre atención posnatal domiciliaria versus hospitalaria, donde la frecuencia de reconsulta del recién nacido que recibió atención posnatal domiciliaria fue del $12 \%(9)$.

En este estudio, las causas de reconsulta más frecuentes en el recién nacido fueron: problemas respiratorios seguidos por ictericia y problemas gastrointestinales, infección y/o sangrado del ombligo y fiebre. Un estudio de alta neonatal precoz y seguimiento inmediato reportó como causas frecuentes de anomalía en el seguimien- 
to del recién nacido hiperbilirrubinemia y problemas respiratorios asociados a obstrucción nasal(4).

Otros estudios también reportan la hiperbilirrubinemia como una de las principales causas de reconsulta para el recién nacido. En un estudio observacional descriptivo se encontró que 74 recién nacidos reconsultaron por ictericia de los cuales 47 habían sido dados de alta tempranamente(10). Otro estudio de evaluación de un programa de seguimiento individualizado del recién nacido dado de alta tempranamente reportó que fue necesario realizar seguimiento y verificación a los recién nacidos que presentaron hiperbilirrubinemia y requirieron seguimiento en diferentes momentos según el nivel de bilirrubina y la necesidad de tratamiento(11). Un estudio de casos y controles realizado en Washington reportó que los recién nacidos tenían más riesgo de reconsulta en los primeros siete días de vida y una de las principales causas de esta reconsulta era la hiperbilirrubinemia(12). Otro estudio realizado en Australia en 2010 reportó que un $91 \%$ de las reconsultas de recién nacidos tuvo a la ictericia como diagnostico principal(13). La ictericia es una de las principales causas de reconsulta debido a que el recién nacido tiene una predisposición a la producción excesiva de bilirrubina por tener un número mayor de glóbulos rojos cuyo tiempo de vida promedio es menor que en otras edades. Además, el sistema enzimático del hígado es insuficiente para la captación y al nacimiento el neonato está expuesto a diferentes traumas que resultan en hematomas o sangrados que aumentan la formación de bilirrubina(14).

Respecto a la madre, la frecuencia de reconsulta después del alta temprana posparto fue de $3,79 \%$ similar a la reportada por Boulvain y Cols, donde la frecuencia fue de $3,5 \%(9)$, y mayor a la del ensayo clínico que reportó una frecuencia de reconsulta de 1,9\% en el grupo de alta temprana y 2,3\% en el grupo control(15). En este estudio, las causas más frecuentes de reconsulta fueron dolor, ardor y/o cólico en zona inferior del abdomen $28,57 \%(\mathrm{n}=8)$, causas no asociadas al parto $28,57 \%(\mathrm{n}=8)$ y mastitis $7,14 \%$ $(\mathrm{n}=3)$. Esto es similar a lo reportado por un estudio acerca de la duración de la estancia hospitalaria y la tasa de reingresos, donde las causas no asociadas al parto fueron la principal causa de reconsulta de la madre, y también los problemas relacionados con la lactancia materna, cólico abdominal, hemorragia posparto y retención de restos placentarios(16).

Aunque en diversos estudios están descritas las principales causas de reconsulta, en los estudios encontrados no se observa una descripción detallada de los casos de reconsulta que requirieron hospitalización ni la mediana de días que duró, tanto para el recién nacido como para la madre. Los análisis realizados donde se determinó la frecuencia de la reconsulta, tanto para el binomio madre-hijo como para el recién nacido, permitieron concluir que los factores asociados son la baja escolaridad de la madre y el alta temprana. Respecto a la asociación de la educación de la madre, el estudio acerca de un seguimiento al recién nacido luego del alta temprana en Sudáfrica reportó también esta asociación; en este, las madres con un nivel educativo de secundaria o superior reportaron un OR de 1,47 IC $95 \%(1,02-2,12)$; es 
decir, mayor probabilidad de no faltar al seguimiento(17).

Relacionado al alta temprana, los estudios revisados de la madre y el recién nacido reportan la frecuencia y sus causas por separado para la madre y el recién nacido, ninguno de ellos analizó el binomio madre-hijo como un conjunto. Dentro de las causas más comunes de reconsulta se encuentra la ictericia. El estudio realizado por Fuerzan y Cols reportó que de 86 recién nacidos que acudieron a consulta, 12 fueron admitidos por ictericia significativa y uno por sepsis precoz; 10 habian egresado entre 25 a 48 horas y 3 egresaron antes de las 24 horas(4).

Así, dado que el alta temprana podría ser un factor relacionado a la reconsulta para el recién nacido, la AAP recomienda el cumplimiento de los siguientes criterios mínimos previos al alta de un recién nacido a término después de una gestación y un parto sin complicaciones: que el curso clínico y el examen físico no revelen anomalías que requieran hospitalización continua, los signos vitales se documenten dentro de los rangos normales, con variaciones apropiadas basadas en el estado fisiológico y estable durante las 12 horas anteriores a la salida; que haya orinado regularmente y haya hecho por lo menos una deposición espontáneamente; que haya completado por lo menos dos comidas exitosas; que no haya evidencia de sangrado excesivo en el sitio de la circuncisión durante al menos dos horas; que se haya determinado la importancia clínica de la ictericia si está presente antes del alta y se hayan realizado planes apropiados de manejo; que el lactante haya sido adecuadamente evaluado y monitoreado para la sepsis; que se disponga de pruebas de laboratorio para la madre y el lactante y se hayan revisado; que haya sido administrada la vacuna inicial contra la hepatitis B, y si la madre no ha sido previamente vacunada, debe recibir toxoide tetánico, toxoide de difteria reducido y vacuna pertussis acelular adsorbida (Tdap) inmediatamente después de que nazca el bebé; que se hayan completado exámenes metabólicos, auditivos y de oximetría de pulmón por protocolo hospitalario y regulaciones estatales; y que se haya brindado información y capacitación a la madre en lactancia materna, micción apropiada y frecuencia de deposición del bebé, cuidados del cordón umbilical, piel y genitales(3).

Para la reconsulta de la madre no fueron encontrados factores asociados. Un estudio prospectivo analítico reportó una tasa de reingreso materno de $0,99 \%$, la cual no aumenta en caso del alta temprana. Además, identificó como principales causas de reconsulta materna problemas con la episiotomía (principalmente hematomas y dolor) y mastitis pero ninguna de estas causas de reconsulta fueron complicaciones graves(18). Esto fue similar a lo encontrado en nuestro estudio.

Dentro de las principales debilidades del estudio está que, debido a que solo $56,3 \%$ mujeres atendieron la llamada de seguimiento, podría haberse presentado sesgo de no respuesta, pero se espera que esta no respuesta no esté asociada a la condición de reconsulta o no reconsulta. Además, se realizó un análisis del total de la muestra que respondió la llamada y reportó un poder del $99 \%$.

También pudo darse el sesgo de memoria durante el seguimiento telefónico dado 
que se realizó después de 10 meses. Sin embargo, los eventos relacionados con la salud de los recién nacidos, son recordados por las madres con mayor precisión. También puede estar presente el sesgo de subjetividad de parte de las madres o el familiar en primer grado que brindó la información durante el seguimiento.

\section{Conclusiones}

Así, se puede concluir que la frecuencia del alta temprana en el binomio madrehijo es determinada por la alta demanda de atenciones maternas perinatales y las instituciones de salud asistenciales. Adicionalmente, el alta temprana no parece ser un factor asociado a la reconsulta de la madre luego del alta posparto, su frecuencia y causas de consulta fueron similares a las reportadas en la literatura y para el recién nacido, y el alta temprana mostró una frecuencia similar a la reportada en algunos estudios además de estar relacionada con la reconsulta. Sin embargo, existen criterios clínicos que pueden ser implementados antes del alta para minimizar el riesgo de reconsulta. Finalmente, las instituciones que prestan servicios de parto deben ajustarse a las recomendaciones de la guía de práctica clínica; pero ante situaciones apremiantes, en las cuales se debe dar alta al binomio antes de las 24 horas, estas deben verificar los factores de riesgo de reconsulta e individualizar los casos.

\section{Agradecimientos}

Un agradecimiento a las madres y recién nacidos que participaron de este estudio e hicieron posible esta investigación y a María Lucia Bunzel por su apoyo en la recolección de los datos.

\section{Referencias}

(1) Parisi VM, Meyer BA. To Stay or Not to Stay? That is the Question. N Engl J Med [Internet]. 1995 Dec 14 [cited 2019 Feb 17];333(24):1635-7. Available from: http://www.nejm.org/doi/abs/ 10.1056/NEJM199512143332412

(2) Brown S, Small R, Argus B, Davis $\mathrm{PG}$, Krastev A. Early postnatal discharge from hospital for healthy mothers and term infants. Cochrane Database of Systematic Reviews Vol. 29, Birth. 2002. p. 291-4.

(3) Cardona-Ospina A, OrdoñezMolina J, Vásquez-Trespalacios EM, Mestra-Palomino L, GarcíaPosada RA. Efectos del alta hospitalaria temprana en el periodo posparto en la Clínica del Prado, Medellín, Colombia. Rev Gerenc y Polit Salud. 2014;13(26):119-26.

(4) Furzán JA. Egreso precoz del recién nacido: [revisión] TT - Early newborn discharge: [review]. Arch venez pueric pediatr [Internet]. 2007;70(3):89-96. Available from: http://www2.scielo.org.ve/ $\mathrm{pdf} / \mathrm{avpp} / \mathrm{v} 70 \mathrm{n} 3 / \mathrm{art} 04 . \mathrm{pdf}$ 
(5) Madden JM, Soumerai SB, Lieu TA, Mandl KD, Zhang F, Ross-Degnan D. Effects of a Law against Early Postpartum Discharge on Newborn Follow-up, Adverse Events, and HMO Expenditures. N Engl J Med [Internet]. 2002 Dec 19 [cited 2019 Feb 17];347(25):2031-8. Available from: http://www.nejm.org/doi/ abs/10.1056/NEJMsa020408

(6) Rite Gracia S, Pérez Muñuzuri A, Sanz López E, Leante Castellanos JL, Benavente Fernández I, Ruiz Campillo CW, et al. Criterios de alta hospitalaria del recién nacido a término sano tras el parto. An Pediatría [Internet]. 2017 May 1 [cited 2018 Apr 18];86(5):289.e1-289.e6. Available from: http://linkinghub. elsevier.com/retrieve/pii/ S1695403316302582

(7) Nacional C, Cinets S. Guías de Práctica Clínica para la prevención , detección temprana y tratamiento de las complicaciones del embarazo , parto o puerperio. 2013.

(8) Rueda, J. D., Otálora, M., \& Rosselli, D. (2015, marzo 28). Implicaciones y Costo-Efectividad del Alta Temprana del Binomio Madre-Hijo Después de un Parto Normal. Medicina, 37(1), 8-16.

(9) Boulvain M, Perneger T V., Othenin-Girard V, Petrou S, Berner M, Irion O. Home-based versus hospital-based postnatal care: A randomised trial.
BJOG An Int J Obstet Gynaecol. 2004;111(8):807-13.

(10) Romero Sánchez J, Romero González J, Picazo Angelín B, Tapia Ceballos L, Díaz Cabrera R, Romero Sánchez I. Alta hospitalaria temprana en recien nacidos a termino sanos. An Esp Pediatr [Internet]. 1999;50(5):479-84. Available from: http://www.scopus . com/inward/record.url?eid= $2-$ s2.0-0345211616\&partner ID $=$ tZOtx3y1

(11) De Carolis MP, Cocca C, Valente E, Lacerenza S, Rubortone SA, Zuppa AA, Romagnoli C. Individualized follow up programme and early discharge in term neonates. Ital J Pediatr. 2014 Jul 15;40:70

(12) Liu LL, Clemens CJ, Shay DK, Davis RL, Novack AH. The safety of newborn early discharge. The Washington State experience. Jama. 1997;278(4):293-8.

(13) Lain SJ, Roberts CL, Bowen JR, Nassar N. Early Discharge of Infants and Risk of Readmission for Jaundice. Pediatrics [Internet]. 2015 Feb 1 [cited 2018 Apr 29];135(2):314-21. Available from: http://www.ncbi.nlm.nih. gov/pubmed/25583922

(14) Sociedad Boliviana de Pediatría. E, Mendieta E, Mir R, Céspedes E, Caballero B. Revista de la Sociedad Boliviana de Pediatría. [Internet]. Vol. 47, Revista de la Sociedad Boliviana de Pediatría. 
Sociedad Boliviana de Pediatriá; 2008 [cited 2018 Apr 24]. 46-52 p. Available from: http: //www.scielo.org.bo/scielo. php?script $=$ sci_arttext\&pid= S1024-06752008000100011

(15) Sainz Bueno JA, Romano MR, Teruel RG, Benjumea AG, Palacín AF, González CA, Manzano MC. Early discharge from obstetricspediatrics at the Hospital de Valme, with domiciliary follow-up. Am J Obstet Gynecol. 2005 Sep;193(3 Pt 1):714-26.

(16) Ford JB, Algert CS, Morris JM, Roberts CL. Decreasing length of maternal hospital stay is not associated with increased readmission rates. Aust $\mathrm{N}$ Z J Public Health [Internet]. 2012 Oct [cited 2018 Apr 29];36(5):430-4. Available from: http://www.ncbi.nlm.nih. gov/pubmed/23025363

(17) Milambo JPM, Cho K, Okwundu C, Olowoyeye A, Ndayisaba L, Chand $\mathrm{S}$, Corden MH. Newborn follow-up after discharge from a tertiary care hospital in the Western Cape region of South Africa: a prospective observational cohort study. Glob Health Res Policy. 2018 Jan 12; 3:2.

(18) Kehila M, Magdoud K, Touhami O, Abouda HS, Jeridi S, Marzouk S Ben, et al. [Early postpartum discharge: outcomes and risk factors of readmission]. Pan Afr Med J [Internet]. 2016 [cited 2018 May 1];24:189. Available from: http://www.ncbi. nlm.nih.gov/pubmed/27795786
(19) Rutas integrales de atención en salud - RIAS [Internet]. [cited 2018 Jun 7]. Available from: https://www .minsalud.gov . $\mathrm{co} / \mathrm{salud} / \mathrm{publica/ssr/Paginas/}$ Rutas-integrales-de-atencion-en-salud-RIAS . aspx 